\title{
Comparative studies of macrophage-biased responses in mice to infection with Toxoplasma gondii ToxoDB \#9 strains of different virulence isolated from China
}

Ai-Mei Zhang ${ }^{1,2+}$, Qian Shen ${ }^{3+}$, Min Li ${ }^{1}$, Xiu-Cai Xu ${ }^{1,2}$, He Chen ${ }^{1}$, Yi-Hong Cai ${ }^{1}$, Qing-Li Luo ${ }^{1}$, De-Yong Chu', Li Yu ${ }^{1}$, Jian Du ${ }^{4}$, Zhao-Rong Lun ${ }^{5}$, Yong Wang ${ }^{6}$, Quan Sha ${ }^{3 *}$ and Ji-Long Shen ${ }^{1^{*}}$

\begin{abstract}
Background: Different from three clonal lineages of Toxoplasma gondii in North America and Europe, the genotype China 1 is predominantly prevalent in China. However, there are different virulent isolates within China 1, such as virulent TgCtwh3 and avirulent TgCtwh6, and little is known about differences in macrophage activation between them. The objective of this study focused on cytokine production, phenotype and markers of activated macrophages, and correlated signaling pathway induced by the two isolates.

Methods: Adherent peritoneal macrophages (termed Wh3-M $\varphi$ and Wh6-M $\varphi$, respectively) harvested from infected mice were cultured for detection of Nitric Oxide and arginase activity, and activated markers on Wh3-M $\varphi /$ Wh6-M $\varphi$ were determined by flow cytometry. In in vitro experiments, the levels of IL-12p40 and TNF-a were measured using ELISA kits, and mRNA expressions of IL-12p40, TNF-a, iNOS, Arg-1 and Ym1 were assayed by real-time PCR. To confirm the activation state of NF-kB p65 in infected cells stained by IF, protein levels of iNOS, Arg-1, Ym1, nuclear NF-KB p65, and phosphorylation of STAT6/STAT3/IKBa were evaluated by Western Blotting. A one-way ANOVA test was used to compare differences among multiple groups.

Results: The result revealed that contrary to the virulent TgCtwh3, the less virulent TgCtwh6 isolate induced a significant increase in IL-12p40 and TNF-a. Although both isolates down-regulated CD80, CD86 and MHCII molecule expression on macrophages, TgCtwh3 promoted up-regulation of PD-L2 and CD206. Wh6-M $\varphi$ generated a high level of NO whereas Wh3-M $\varphi$ up-regulated $\mathrm{Ym} 1$ and arginase expression at transcriptional and protein levels. In terms of signaling pathway, TgCtwh3 induced phospho-STAT6, conversely, TgCtWh6 led to NF-kB p65 activation. Conclusions: The virulent TgCtwh3 isolate induced macrophages to polarize toward alternatively activated cells with STAT6 phosphorylation, whereas the less virulent TgCtwh6 elicited the development of classically activated macrophages with nuclear translocation of NF-KB p65. This discrepancy suggests that it is necessary to thoroughly analyze the genotype of TgCtwh3 and TgCtwh6, and to further study other effector molecules that contribute to the macrophage polarization in T. gondii.
\end{abstract}

Keywords: Toxoplasma gondii, Genotyping, Macrophages, Macrophage activation, Signaling pathway

\footnotetext{
*Correspondence: qsha2@yahoo.com; shenjilong53@126.com

${ }^{\dagger}$ Equal contributors

${ }^{3}$ Department of Immunology, Anhui Medical Universty, Hefei, Anhui, China

${ }^{1}$ Anhui Provincial Laboratories of Pathogen Biology and Zoonoses,

Department of Microbiology and Parasitology, Anhui Medical University,

Hefei, Anhui, China

Full list of author information is available at the end of the article
} waiver (http://creativecommons.org/publicdomain/zero/1.0/) applies to the data made available in this article, unless otherwise stated. 


\section{Background}

Toxoplasma gondii is an intracellular parasite capable of infecting a broad spectrum of hosts including up to $30 \%$ of the world's human population [1]. Infection occurs when individuals ingest undercooked meat containing cysts of the parasite or consume food contaminated with oocysts. Although most infections are mild in healthy adults, life-threatening consequences may develop in immunocompromised individuals and in utero infection can lead to major defects in the fetus [2].

During acute infection, tachyzoites, the rapidly replicative form of the parasite, elicits an extremely strong type Th1 immune response, characterized by proinflammatory cytokine production such as IL-6, IFN- $\gamma$ and TNF- $\alpha$. Macrophages can provide a niche permissive for parasite replication and are the most abundant cell type infected by Toxoplasma [3]. It has been clarified that macrophages can be phenotypically polarized by the micro-environment to mount specific functional programs [4]. Polarized macrophages have been classified in two main clades: classically activated macrophages (or M1) whose typical activating stimuli are IFN- $\gamma$ and LPS, and alternatively activated macrophages (or M2) induced by exposure to IL-4 and IL-13. M1 exhibits antimicrobial functions against intracellular pathogens which is conducted by the production of reactive oxygen and nitrogen intermediates such as $\mathrm{NO}$ and promote strong Th1 responses, while M2 is accompanied by diminished proinflammatory cytokine secretion and shares high expression of scavenger, mannose (CD206) and galactose receptors [5].

Recent work on $T$. gondii polymorphism-associated immune responses show that mouse macrophages infected with type I and III strains are polarized toward an M2 activation state through activation of STAT6, while type II infected macrophages are M1-like cells elicited by activation of NF-kB $[3,6]$. Type I and type III rhoptry kinase ROP16, inferred as one of virulence determinants, can constitutively activate STAT6, and type II dense granule antigen GRA15 is responsible for strain-specific NF- $\kappa$ B activation [6].

The majority of $T$. gondii strains isolated from humans and animals in North America and Europe have been grouped into three predominant clonal lineages (types I, II and III) that differ genetically by less than $1 \%[7,8]$. Regardless of the genetic background of mice, type I strains are highly virulent with an LD100 $=1$, whereas type II or type III strains associate with host genetic background and display lower virulence with an LD50 $\approx 10^{2}$ and $\approx 10^{5}$, respectively [9]. Recently there were several studies revealing that a few major clonal lineages of $T$. gondii dominate in different geographical regions. For example, the type 12 lineage is most common in wildlife in North America [10], and the Africa 1 and 3 are among the major types in Africa [11]. Our previous study and other lab research showed that the genotype China 1 (ToxoDB \#9) is dominantly prevalent in China [12-15].

The outcome of toxoplasmosis in the mouse model is mainly influenced by the genotype of strain of $T$. gondii [16]. Interestingly, within the genotype China 1, isolates of TgCtwh3 and TgCtwh6 have remarkably different virulence to mice [12], in which TgCtwh3 is highly virulent and cause lethal infection, whereas TgCtwh6 is mildly virulent and able to establish latent infection with cysts in the brain of mice. This virulent difference triggered our interest in understanding the distinction between the two isolates. In the present study, we systematically compared the acute immune response of host, macrophage activation and modulation of the signaling pathway to infection with TgCtwh3 and TgCtwh6 tachyzoites respectively.

\section{Methods}

\section{Ethics statement}

All animal experiments were conducted in strict accordance with the Chinese National Institute of Health Guide for the Care and Use of Laboratory Animals (1998) and approved by the Institutional Review Board of Anhui Medical University Institute of Biomedicine (Permit Number: AMU26-081108). All efforts were made to minimize the animal's suffering.

\section{Mice and parasites}

Six- to eight-week old BALB/C mice were purchased from the Animal Department of Anhui Medical University and acclimatized for at least 1 week before the experiment. Mice were bred in-house under specific pathogen-free conditions with free access to food and water.

The tachyzoites of mouse-virulent TgCtwh3 isolates or $\mathrm{RH}$ strain were harvested from the mouse peritoneal exudates by injection of ice-cold D-Hanks solution on day 3 after infection, and then isolated by centrifugation at $35 \times \mathrm{g}$ for $5 \mathrm{~min}$ to discard contaminating host cells. After the supernatant was centrifuged at $1350 \times \mathrm{g}$ for $10 \mathrm{~min}$, the parasites were washed once and maintained by serial passages in L929 fibroblast monolayers for further infection experiments in vitro and in vivo.

The tachyzoites of TgCtwh 6 or PRU strain were initially obtained by inoculating brain homogenate containing cysts from infected mice and then cultured with L929 fibroblasts. For initial passages, fibroblast monolayers were detached by scraping, and cells were forced through a 27-gauge needle to release the intracellular parasites. The tachyzoites of TgCtwh 6 and PRU were maintained by continual cell passages.

\section{Cell culture}

The mouse macrophage RAW 264.7 cell line was maintained in DMEM (GIBCO) supplemented with 10\% 
heat-inactivated fetal calf serum (FCS, GIBCO), $100 \mathrm{U} / \mathrm{ml}$ penicillin, and $0.1 \mathrm{mg} / \mathrm{ml}$ streptomycin. Bone marrowderived macrophages (BMM $\phi s)$ were generated by culturing bone marrow cells isolated from femurs and tibia of 3-6 months old female Swiss Webster mice in 20\% L929 cell-conditioned medium, as previously described [17]. Used as a source of macrophage colony-stimulating factor, L929-conditioned medium was prepared by harvesting supernatants from L929 murine fibroblasts cultured for 6-7d in RPMI 1640, supplemented with antibiotics and FCS as indicated above. All parasite strains and cell lines were routinely checked for mycoplasma contamination, and remained negative throughout the experiments.

\section{In vivo and in vitro infection assays}

For in vivo experiment, $1 \times 10^{4}$ tachyzoites of TgCtwh3 or TgCtwh6 were inoculated i.p. into mice. After 3 days of infection, mice were euthanized and peritoneal exudate cells (PECs) were harvested by washing the peritoneal cavity with $10 \mathrm{ml}$ of ice-cold D-Hanks solution. PECs were cultured in RPMI 1640, supplemented with $10 \%$ FCS, $100 \mathrm{U} / \mathrm{ml}$ penicillin, and $0.1 \mathrm{mg} / \mathrm{ml}$ streptomycin. Three hours later nonadherent cells were washed off and the remaining adherent macrophages $\left(5 \times 10^{6}\right.$ cells per well) (termed Wh3-M $\phi$ and Wh6-M $\phi$, respectively) were cultured in 6-well plates for $12 \mathrm{~h}$.

For in vitro infection assays, maintained in 12-well plates $\left(1 \times 10^{6}\right.$ cells per well), BMM $\phi$ s and RAW 264.7 cells were pre-stimulated for $24 \mathrm{~h}$ with $100 \mathrm{ng} / \mathrm{ml} \mathrm{re-}$ combinant murine IFN- $\gamma$ (PrimeGEne, China) and $100 \mathrm{ng} / \mathrm{ml}$ LPS (Sigma, USA), or with $5 \mathrm{ng} / \mathrm{ml} \mathrm{rMu} \mathrm{IL-13}$ plus $20 \mathrm{ng} / \mathrm{ml}$ IL-4 (PrimeGEne), or left unstimulated, then infected with freshly lysed $T$. gondii tachyzoites at a parasite to host cell ratio of 2:1. Cells were incubated for an additional $24 \mathrm{~h}$.

\section{Measurement of cytokine levels}

After in vitro culture for $24 \mathrm{~h}$, supernatants from $\mathrm{RH}-$, PRU-, TgCwh3- or TgCwh6-infected BMM $\phi$ s were collected and frozen at $-80^{\circ} \mathrm{C}$. The concentration of IL-12p40 and TNF- $\alpha$ was determined using ELISA kits (Uscnk, USA and MultiSciences, China, respectively) following the manufacturer's directions.

\section{Determination of Nitric Oxide (NO) production and arginase activity}

Cell culture supernatants from Wh3-M $\phi$, Wh6-M $\phi$ or normal peritoneal $\mathrm{M} \phi$ were collected at $12 \mathrm{~h}$, and supernatants from $\mathrm{TgCwh} 3-$ or TgCwh6-infected BMM $\phi$ s or RAW 264.7 cells and uninfected control cells were collected at $24 \mathrm{~h}$ after infection. Nitric oxide production was assessed by nitrite accumulation in the culture media using the Griess Reagent System (Promega, USA).
Briefly, $50 \mu \mathrm{l}$ supernatant or standard solution (1.56$100 \mu \mathrm{m}$ sodium nitrite) were incubated in duplicate with $50 \mu \mathrm{l}$ of sulfanilamide solution for 5-10 min, then incubated with $0.1 \% \mathrm{~N}$-1-naphthyldiamine dihydrochloride for $5 \mathrm{~min}$ and the OD was measured at $540 \mathrm{~nm}$.

Arginase activity was measured in macrophage lysates by colorimetric method as previously described [18], and defined as the amount of urea produced from total protein of macrophages.

\section{Flow cytometry}

After 3 days of TgCtwh3 or TgCtwh6 infection, PECs were harvested and cultured in RPMI 1640 as described above. Three hours later adherent $\mathrm{Wh} 3-\mathrm{M} \phi$ and $\mathrm{Wh} 6-\mathrm{M} \phi$ were collected, washed twice with PBS containing 3\% FCS and labeled with the antibodies of interest at the appropriate dilution for $20 \mathrm{~min}$ at $4^{\circ} \mathrm{C}$ in the dark. The antibodies include FITC-conjugated anti-F4/80, PE-Cy5-conjugated anti-MHCII, PE-conjugated PD-L2 (programmed death ligand, PD-L) (eBioscience, USA), PE-conjugated PD-L1, PECy5-conjugated anti-CD80, APC-conjugated anti-CD86 (BD Pharmingen, USA), Alexa Fluor 647-conjugated anti-CD206 (BioLegend, USA), as well as appropriate isotype control antibodies. Uninfected macrophages were set up in parallel. Cells were washed three times in FACS buffer and fixed in $2 \%$ paraformaldehyde before acquisition and analysis (FACSCanto $^{\mathrm{m}}$, BD Biosciences and FlowJo software 7.6).

\section{RT-PCR analysis}

By resuspension in TRIzol reagent (Invitrogen, CA) RNA was prepared from $\mathrm{TgCwh} 3$ - or $\mathrm{TgCwh} 6$-infected BMM $\phi$ s at $24 \mathrm{~h}$ postinfection. Total RNA was extracted and cDNA was synthesized using the RevertAid First Strand cDNA Synthesis Kit (Fermentas, USA) following the manufacturer's instructions. Relative quantification of the genes of interest was measured by real-time PCR using the SYBR Premix Ex Taq ${ }^{\text {Tax }}$ (TaKaRa, Dalian, China). The primers (synthesized by Shenggong, Shanghai, China) for amplification of gene fragments are listed in Table 1. Only experiments, in which a distinct single peak was observed with a melting temperature different from that of the notemplate control, were analyzed. Real-time PCR of the housekeeping gene GAPDH allowed normalization of the expression of the interested genes and expression relative to non-infected control samples was calculated utilizing the $\Delta \Delta C_{\mathrm{T}}$ method. Reactions were carried out in an Applied Biosystems 7500 Fast Real Time PCR System (Applied Biosystems, SA). Experiments were performed in triplicate, and mRNA fold change represents $2^{-\Delta \Delta C T}$.

\section{Western blotting}

$\mathrm{BMM} \phi \mathrm{s}$ grown in a 6-well plate were infected with TgCtwh3 and TgCtwh6 parasites respectively (2:1 ratio of parasites to $M \phi)$ for $24 \mathrm{~h}$. After being washed with 
Table 1 Sequences of oligonucleotide primers used for real-time PCR

\begin{tabular}{|c|c|c|c|}
\hline Target & Forward primer $\left(5^{\prime}-3^{\prime}\right)$ & Reverse primer $\left(5^{\prime}-3^{\prime}\right)$ & Length(bp) \\
\hline TNF-a & GAACTTCGGGGTGATCGGTC & TGTCTITGAGATCCATGCCG & 221 \\
\hline IL-12p40 & TGCCAGGAGGATGTCACCT & GGCGGGTCTGGTTTGATGAT & 137 \\
\hline iNOS & GTTCTCAGCCCAACAATACAAGA & GTGGACGGGTCGATGTCAC & 127 \\
\hline Arginase-1 & CTCCAAGCCAAAGTCCTTAGAG & AGGAGCTATCATTAGGGACATC & 185 \\
\hline Ym1 & AGAAGGGAGTTTCAA ACCTGGT & GTCTTGCTCATGTGTGTAAGTGA & 109 \\
\hline GAPDH & CAACTITGGCATTGTGGAAGG & ACACATTGGGGGTAGGAACAC-3' & 224 \\
\hline
\end{tabular}

ice-cold PBS, in the presence of Protease/Phosphatase Inhibitor Cocktail (Cell Signaling, USA) infected cells were lysed by addition of lysis buffer, or nuclear proteins were extracted according to manufacturer's instructions (NucBuster Protein Extraction Kit, Novagen, German). Then total cell lysates or nuclear proteins were subjected to $8 \%$ SDS-PAGE respectively. Proteins were transferred to a nitrocellulose membrane (Millipore Corp, Billerica, MA), which was blocked in TBS/0.1\% Tween-20/5\% non-fat dry milk and incubated with primary antibodies. The primary antibodies applied included: anti-iNOS and anti-Stat6 (BD Transduction Laboratories, USA), anti-Ym1 (Stemcell Technologies, Canada), anti-arginase 1 (sc-20150, CA), anti-NF-kB p65 (sc-8008), anti-IkB $\alpha$ (sc-847), anti-p-IкB $\alpha$ (sc-8404), anti-Stat6 (pY641, BD PharMingen), anti-T. gondii SAG1 (ViroStat, Portland), anti-Stat3 (4904p), anti-pStat3 (9145P) and anti- $\beta$-actin antibody (Cell Signaling). After being washed, membranes were incubated with appropriate horseradish peroxidase-conjugated secondary antibodies (Promega, USA). The bound antibodies were visualized by enhanced chemiluminescence using ECL kit (SupreSignal West Pico; Thermo Scientific), and the results were analyzed with ImageJ software.

\section{Immunofluorescence staining}

TgCtwh3 or TgCtWh6 parasites were allowed to invade cells on coverslips and incubated for different time points. Then coverslips were washed twice in PBS and fixed for $20 \mathrm{~min}$ at room temperature with $4 \%$ paraformaldehyde. After being washed the cells were permeabilized with $0.3 \%$ Triton X-100 for $10 \mathrm{~min}$, and blocked in goat serum for $1 \mathrm{~h}$. To determine the nuclear or cytoplasmic NF-kB p65 expression, HeLa cells were simultaneously incubated with anti-NF-кB p65 antibody (ab7970, UK) along with anti-T. gondii p30-FITC (6203, ViroStat, Portland) in the dark overnight at $4^{\circ} \mathrm{C}$. Coverslips were washed three times in PBS, incubated for $1 \mathrm{~h}$ at room temperature with tetramethylrhodamine goatanti-rabbit IgG (H+ L) (T2769, Invitrogen), and then incubated with Hoechst dye for 5 min for DNA visualization. Finally, coverslips were extensively washed and mounted on a glass slide with anti-fading agent. Cell preparations were examined with an inverted fluorescence microscope (Olympus IX51), and photos were taken using a digital camera (Nikon DS-5M) and NIS-Elements software F3.0.

\section{Statistical analysis}

Data are expressed as mean \pm SD. A one-way ANOVA test (Dunnett test) or Student's $t$-test were used to compare differences among multiple groups or between two unpaired samples, respectively. Differences were considered statistically significant at $P<0.05$.

\section{Results}

TgCtwh3 and TgCtwh6 strains display different virulence Our previous study showed that different from the three clonal lineages of $T$. gondii strains isolated in North America and Europe, the genotype China 1 (ToxoDB\#9) is a major lineage prevalent in China with different virulent strains [12]. TgCtwh3 strain leads to fatal infection while mice innoculated with TgCtwh6 can establish latent infection. To evaluate acute virulence, mice were challenged i.p. with low (500 tachyzoites) or high $\left(5 \times 10^{5}\right.$ tachyzoites) doses of the TgCtwh3 or TgCtwh6 isolates. It was found that mice inoculated with $500 \mathrm{TgCtwh} 3$ tachyzoites uniformly succumbed to infection within 9 days (Figure 1A), in contrast, no death was noted in animals given an equivalent dose of TgCtwh6 tachyzoites during the whole experiment. When infected with $5 \times 10^{5}$ TgCtwh 6 tachyzoites, $20 \%$ of the mice survived beyond 30 days and cysts could be found in the brains. Additionally, TgCtwh3 tachyzoites had a higher proliferation (Figure 1B, 1C, 1D) and completely lysed a flask of cultured cells much faster than TgCtwh6 tachyzoites in vitro (Figure 1E).

\section{Distinct cytokine profile of macrophages infected with TgCtwh3 and TgCtwh6 isolates}

To examine the production of cytokines that are associated with $\mathrm{M} 1 / \mathrm{M} 2, \mathrm{BMM} \phi$ s were challenged with tachyzoites of TgCtwh3 and TgCtwh6. The data indicated that TgCtwh6 isolate, rather than TgCtwh3, induced a significant increase of IL-12p40 and TNF- $\alpha$ (Figure 2A, 2B). In concert with protein levels, $\mathrm{BMM} \phi \mathrm{s}$ infected with TgCtwh6 also showed elevated mRNA expression of IL12 p40 and TNF- $\alpha$ (Figure 2C, 2D).

TgCtwh3 and TgCtwh6 down-regulated CD80, CD86 and MHCII molecule expression on macrophages

Several studies showed that macrophages infected with T. gondii differently regulated the levels of MHC II and 


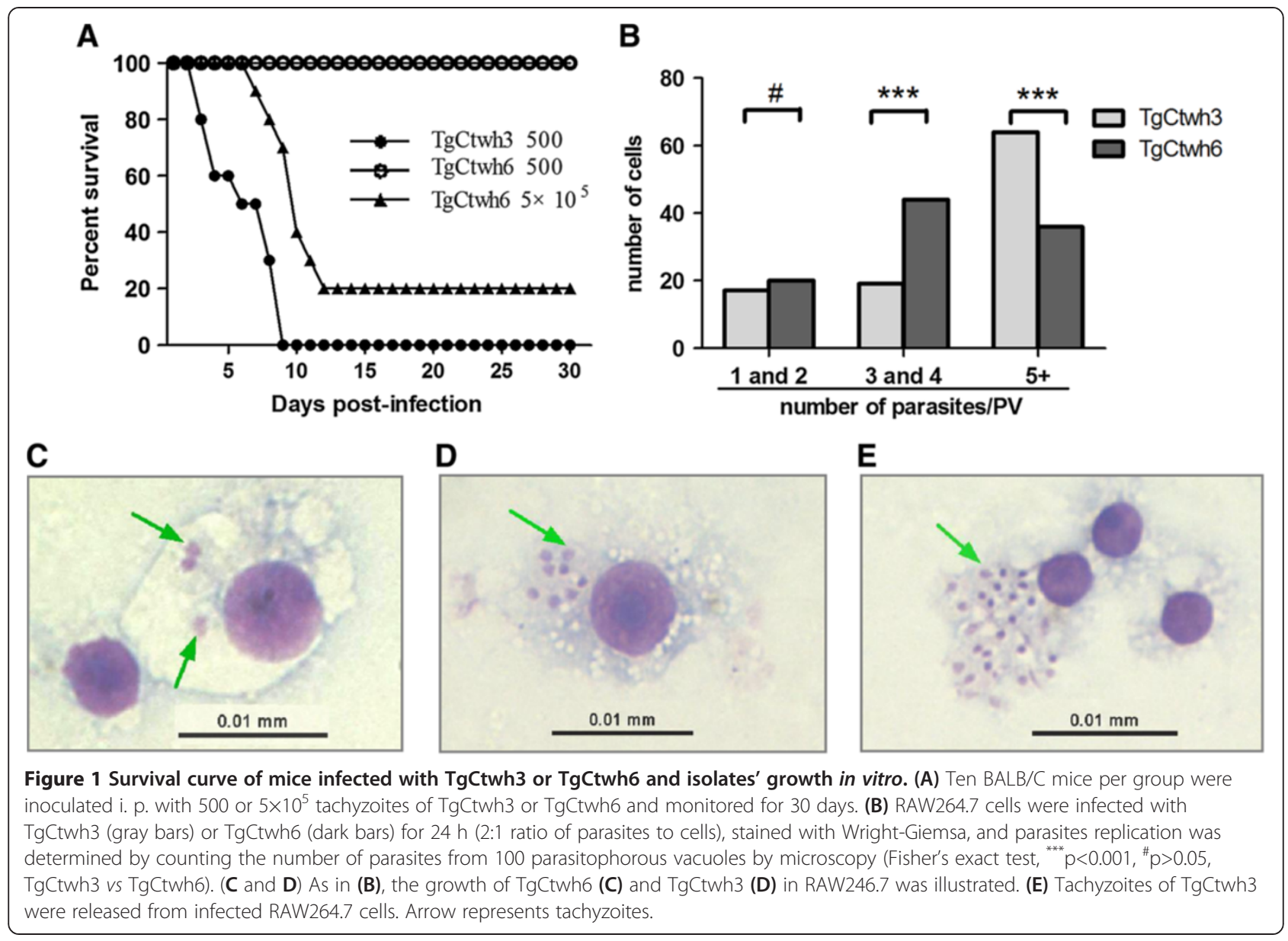

co-stimulatory molecules [19-21]. Since the genotype China 1 differs from the archetypal I, II and III strains, the surface expression of CD80, CD86 and MHC class II molecules on peritoneal macrophages of infected mice was quantified by flow cytometry (Figure 3A). The results indicated that both Wh3-M $\phi$ and Wh6-M $\phi$ expressed lower F4/80 than uninfected macrophages, and there was no significant difference in mean fluorescence intensity (MFI) for F4/80 on Wh3-M $\phi$ vs Wh6$\mathrm{M} \phi$ (Figure 3C). Additionally, neither Wh3-M $\phi$ nor Wh6-M $\phi$ could up-regulate the expression of CD80, CD86 and MHC class II molecules (Figure 3D).

\section{Wh3-M $\varphi$ up-regulated PD-L2 and CD206 whereas Wh6-M $\varphi$} modestly expressed PD-L1

To determine the activation state of macrophages, the expression of CD206, PD-L1 and PD-L2 on peritoneal macrophages of mice was investigated. The results revealed that TgCtwh3 was able to strongly promote PD-L2 expression and Wh3-M $\phi$ was stained positive for CD206, with MFI being $112.78 \pm 19.91$ and $105.32 \pm 25.59$, respectively (Figure 3B, 3E). Therefore, Wh3-M $\phi$ displayed features similar to alternative activated macrophages (M2). In contrast, relative to resident peritoneal macrophages, Wh6-M $\phi$ modestly up-regulated the expression of PD-L1 and PD-L2 with MFI being $139.83 \pm 20.82$ vs $27.86 \pm 4.88$ $(\mathrm{p}<0.001)$ and $37.48 \pm 10.34$ vs $5.97 \pm 2.96(\mathrm{p}<0.01)$, respectively (Figure 3B, 3E).

\section{Wh6-M $\varphi$ generated high level of NO whereas Wh3-M $\varphi$ up-regulated $\mathrm{Ym} 1$ and arginase expression}

To further explore the phenotypic features of activated macrophages infected by TgCtwh3 or TgCtwh 6 strains, a variety of in vitro and in vivo assays were performed on $\mathrm{NO}$ and arginase activity, expression of inducible nitric oxide synthase (iNOS), arginase-1 (Arg-1) and Ym1 at transcription and protein levels. As a result, Wh6-M $\phi$ gave rise to robust production of $\mathrm{NO}$ which was 14-fold higher than uninfected $M \phi$ (Figure 4A), while TgCtwh3 infection elicited high arginase activity in macrophages as measured by the production of urea (Figure 4B).

To verify the in vivo observations, BMM $\phi$ s or RAW 264.7 cells were infected with either TgCtwh3 or TgCtwh6 isolates for $24 \mathrm{~h}$ and the arginase activity was measured. The results indicated that BMM $\phi s$ or RAW 264.7 cells infected with TgCtwh3, rather than TgCtwh6, 


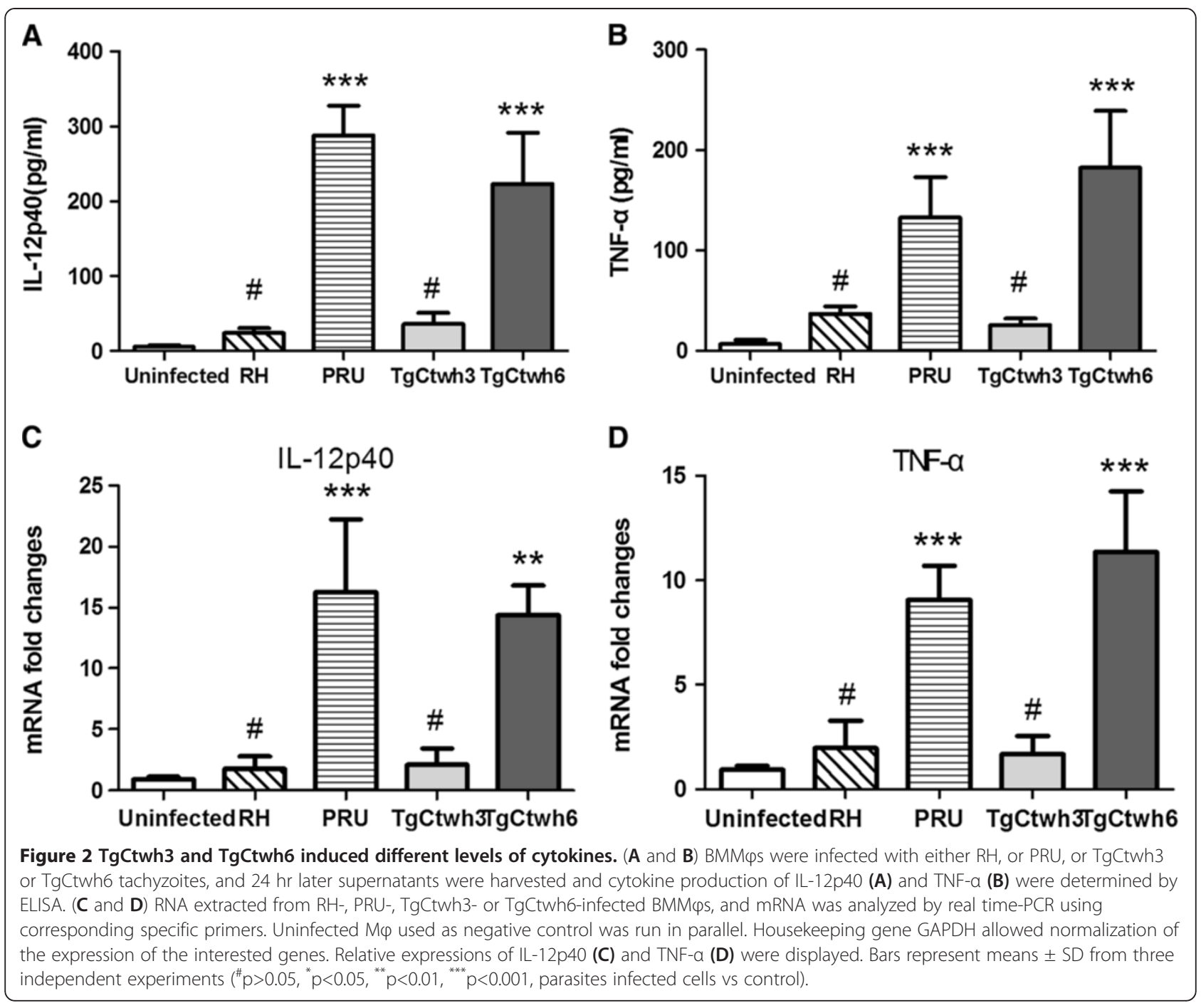

had high arginase activity (Figure $4 \mathrm{C}$ ). To test gene profiles, which are associated with the M1 or M2 phenotypes, the total RNA was extracted from infected BMM $\phi$ s at $24 \mathrm{~h}$ postinfection and the transcriptional response was analyzed by real time PCR (Figure 4D). Consistent with the elevated arginase activity stated above, $\mathrm{BMM} \phi \mathrm{s}$ infected with TgCtwh3 displayed a high level of Arg-1 mRNA, 8-fold higher than that with TgCtwh6. The transcription level of Ym1, which encodes a chitinase-like secretory lectin, were also remarkably upregulated in TgCtwh3 infected BMM $\phi$ s. In terms of iNOS, TgCtwh6 elicited a marked increase of mRNA compared to uninfected macrophages, which was correlated to the NO production.

Cell lysates collected from BMM $\phi$ s infected with TgCtwh3 or TgCtwh6 were subjected to Western blotting in order to assay the protein expression of iNOS, Arg-1 and Ym1. Uninfected and RH- (type I), PRU(type II) infected BMM $\phi$ s were run in parallel as control.
The data showed that $\mathrm{RH}$ infection elicited robust Ym1 and high Arg-1 expression but $\mathrm{NO}$ was undetectable (Figure 4E, 4F), in contrast to low Ym1 on PRU-infected macrophages. BMM $\phi$ s infected with TgCtwh3 shared the features of high Arg-1 and Ym1 expression, which were similar to those with RH strain, while TgCtwh6 infection induced strong iNOS expression (Figure 4E, 4F).

\section{High expression of arginase in M2 is associated with parasite proliferation}

To evaluate the effects of M1 or M2 on intracellular parasites, RAW 264.7 cells, pretreated either with IL-4 plus IL-13 or IFN- $\gamma$ plus LPS, were infected with TgCtwh3 and TgCtwh6 respectively, and assayed for arginase activity and parasite growth. In general, arginase activity was enhanced in IL-4 and IL-13-treated cells, especially in those co-infected with TgCtwh3 (Figure 5A). All RAW 264.7 cells, when pre-exposed to IFN- $\gamma$ and LPS, produced no or less urea. Moreover, the increased 


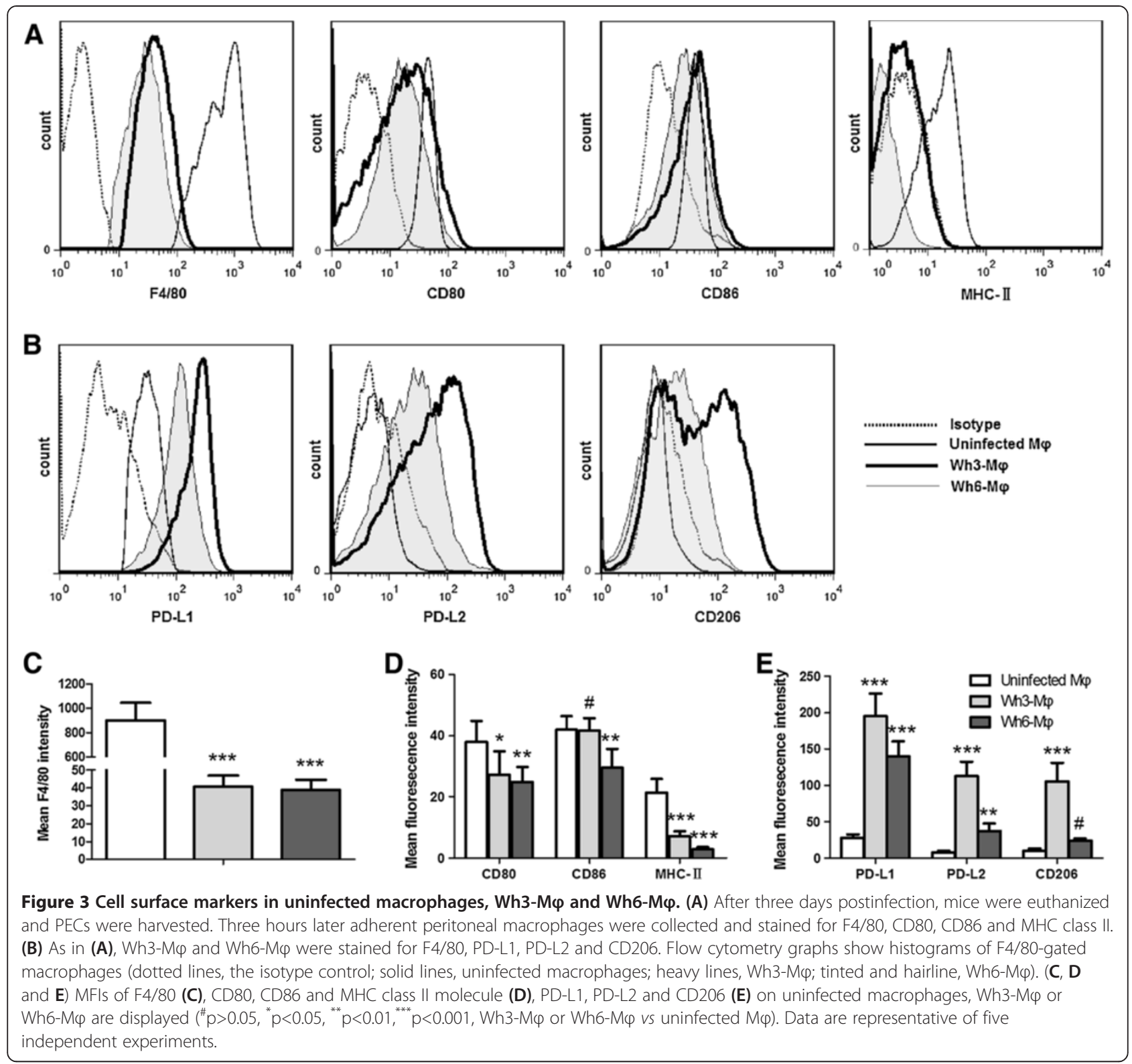

proliferation of TgCtwh3 in IL-4 and IL-13-treated cells was also obvious by counting the number of parasites per vacuole (Figure 5B-5E).

TgCtwh3 induced phospho-STAT6 whereas TgCtWh6 led to activation and nuclear translocation of NF-KB p65

To observe nuclear translocation of the NF- $\mathrm{kB}$ p 65 subunit, HeLa cells were infected with either TgCtwh3 or TgCtwh6 and stained by immunofluorescence (IF). It was found that the cells infected with TgCtwh6 showed high levels of NF-kB p65 in the nuclei, whereas no translocation or only low levels of NF-kB p65 could be noted in the nuclei induced by TgCtwh3 (Figure 6A, 6B).

To further determine the signaling pathway mediated by the isolates of genotype China 1, we preformed
Western blotting with total lysates or nuclear lysates from BMMфs infected with either TgCtwh3 or TgCtwh6. Our results showed that, similar to $\mathrm{RH}$, TgCtwh3 induced strong phosphorylation of STAT6 and STAT3, whereas TgCtwh6 triggered less phosphorylated STAT6 and merely moderate activation of p-STAT3 (Figure 6C, 6E). Moreover, results from Western blotting with nuclear lysates demonstrated that, resembling PRU strain, TgCtwh6 tachyzoites were capable of causing the phosphorylation and nuclear translocation of NF-kB p65 subunit (Figure 6D, 6G), which was correlated with the results from IF. By contrast, this response was significantly attenuated in virulent TgCtwh3 tachyzoites.

Since the phosphorylation and degradation of an inhibitory protein IкB $\alpha$ triggers the nuclear translocation 


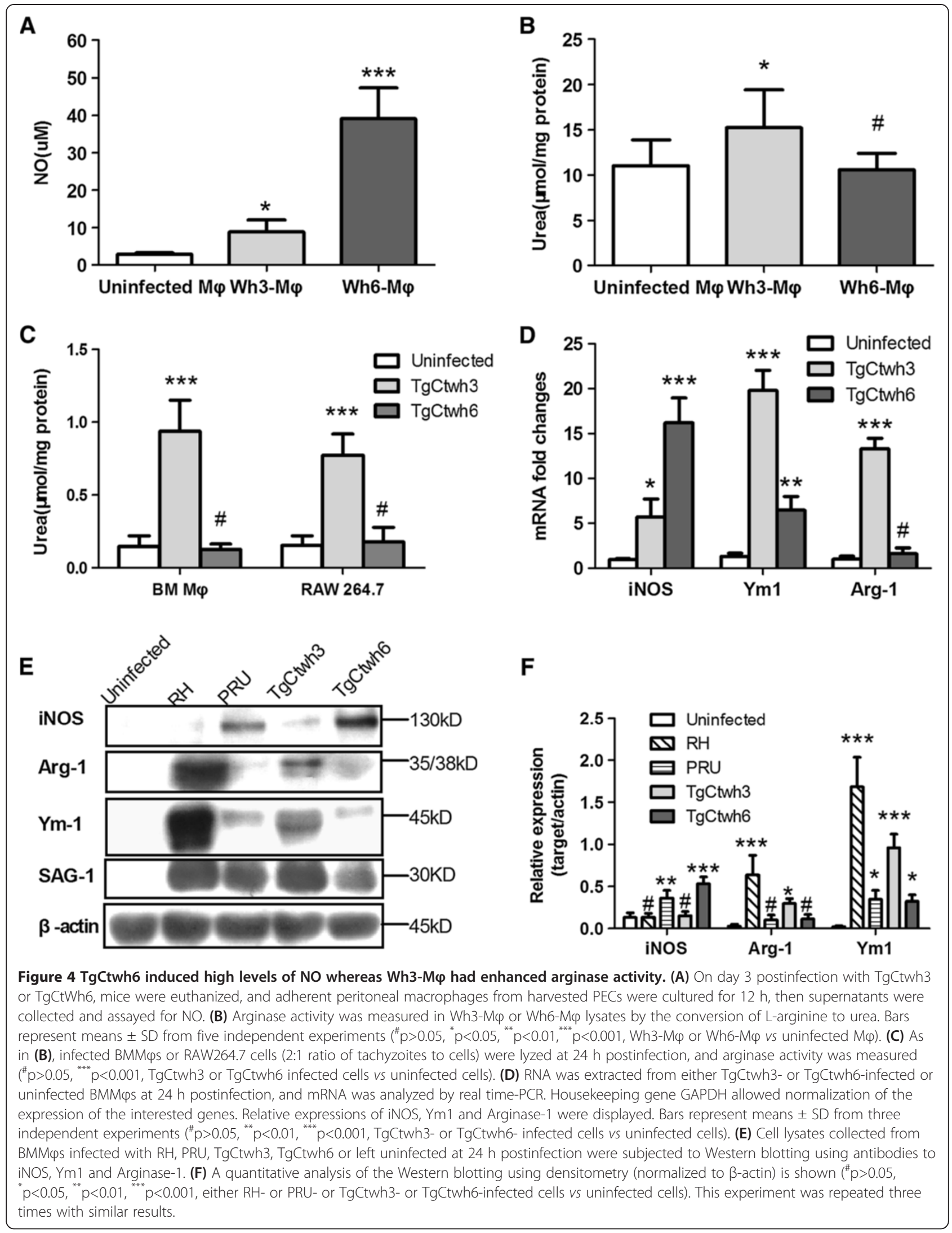




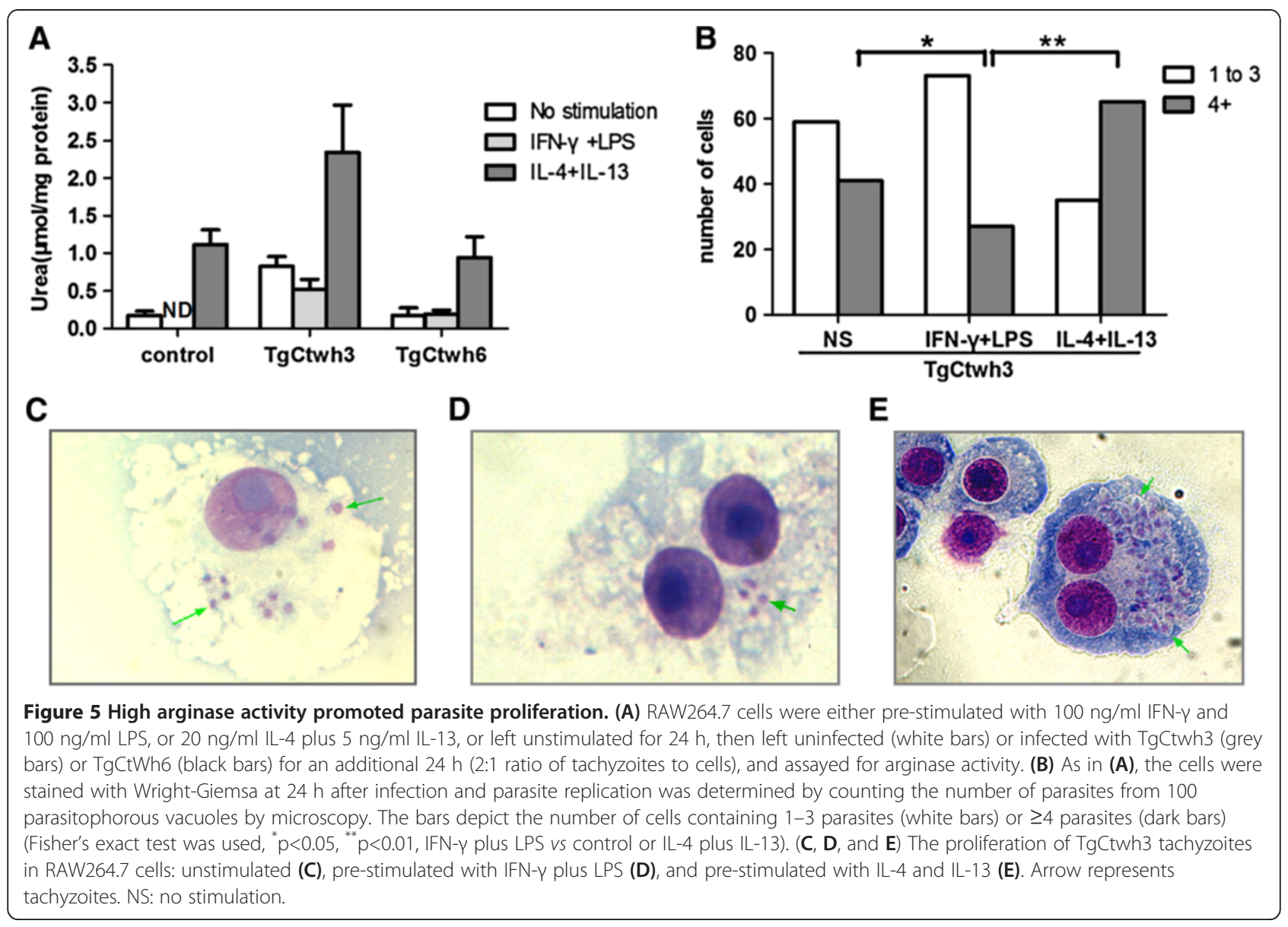

of NF- $\mathrm{kB}$ transcription factor, the expression of phospho-IкB $\alpha$ was also detected. In parallel with nuclear NF-kB p65, the level of phospho-IкB $\alpha$ in cells infected with TgCtwh6 was obviously higher than in cells infected with TgCtwh3 (Figure 6D, 6F).

\section{Discussion}

Recently we have identified several genotypes of Toxoplasma isolates from humans and animals, among them the type China 1 is a dominant clonal lineage prevalent in China $[12,14,22]$. The present study showed that TgCtwh3 and TgCtWh6 strains dramatically differ in virulence to mice and trigger macrophage-biased activation. Here we found that virulent TgCtwh3 isolate induces the alternatively activated macrophage (M2) phenotype characters by presenting high arginase expression and activity, and STAT6 activation associated with low-level IL-12p40 production. By contrast, less virulent TgCtwh6 isolate activates macrophages similar to classically activated macrophages (M1) with phosphorylation of NF-kB p65 and high secretion of IL-12p40.

Similar to earlier findings that avirulent $T$. gondii strain NTE could down-regulate the expression of MHC class II molecules [20], the present results revealed that both Wh3-M $\phi$ and Wh6-M $\phi$ were completely negative for induction of MHC-II expression (Figure 3A, 3D). Additionally, neither Wh3-M $\phi$ nor Wh6-M $\phi$ could upregulate CD80 or CD86 expression, which differs from the reports stating that both molecules, or only CD80/ CD86 expression, were up-regulated in Toxoplasmainfected cells $[19,23]$. This discrepancy might be the result of different cell types and parasite genotypes used in the studies. It is presumed that down-regulation of CD80 and CD86 along with negative expression of MHC class II molecules might represent a straightforward strategy for China 1 isolates to attenuate the interaction of parasitised antigen presenting cells with specific CD4+ T lymphocytes, as long as the infected cells express insufficient amounts of MHC class II molecules and co-stimulatory signals to stimulate $\mathrm{T}$ cell activation.

In addition to expression of CD206, PD-L2 (Figure 3B) and high arginase activity (Figure 4B, 4C), TgCtwh3infected macrophages possess traits similar to M2, such as up-regulation of Arg-1 and $\mathrm{Ym} 1$ at both transcription and protein levels (Figure 4D, 4E). Just as Murry [24] speculated that $T$. gondii I/III strains induce M2 activation bypassing the requirement for exogenous IL-4 and IL-13, it might be unlikely that endogenous IL-4 participates in 


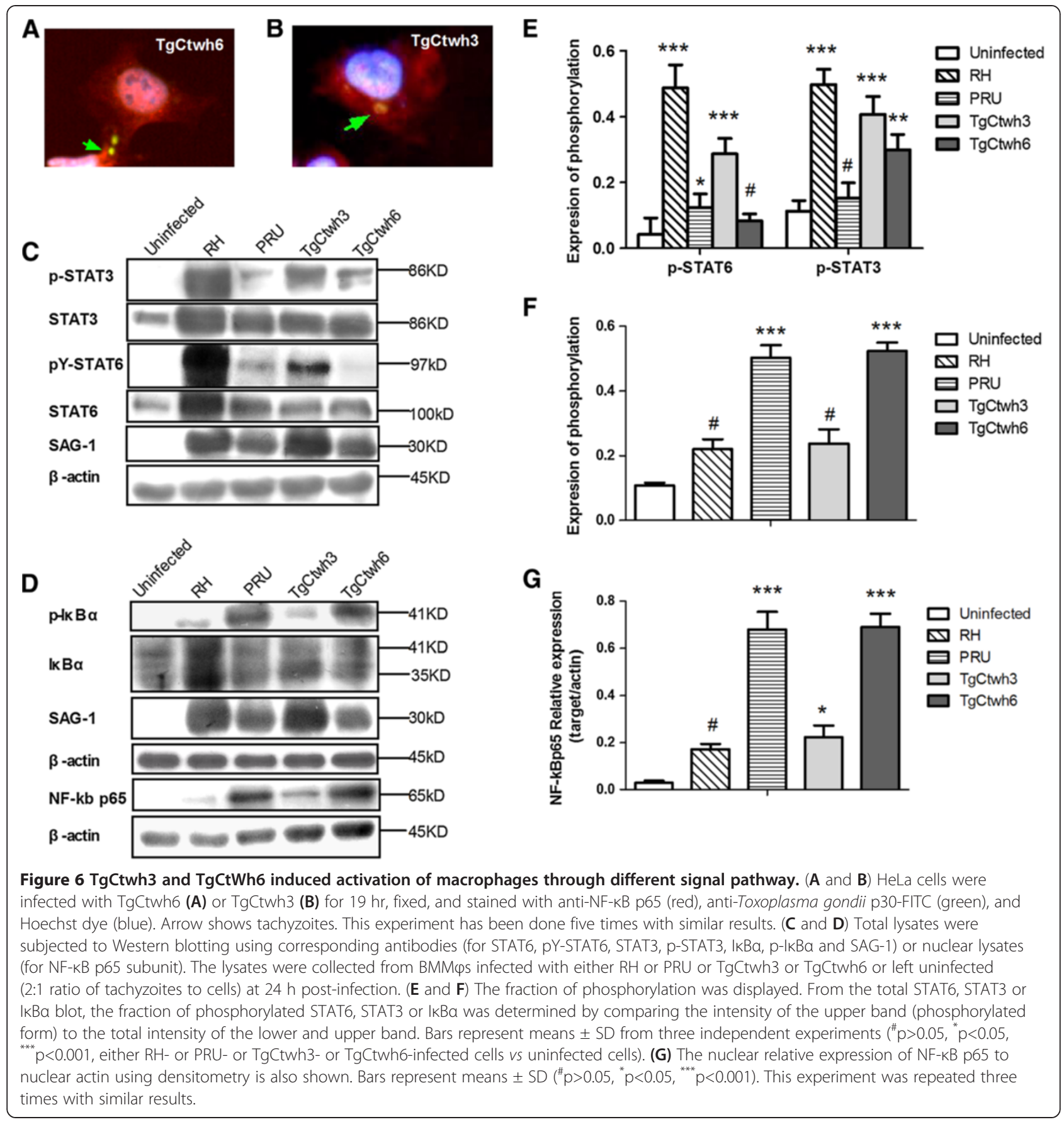

the skewing of TgCtwh3-infected macrophages, since no IL-4 production was detectable in the supernatants (data not shown). Moreover, it is likely that at an early time of infection, with the activation of M2, the induction of Arg-1 could be exploited by TgCtwh3 to promote its growth as described previously (Figure 5) [25,26]. Early low levels of IL-12 and TNF- $\alpha$ (Figure 2) [27] and migratory dendritic cells and macrophages hijacked by $T$. gondii for parasite dissemination [28] may attribute to later high parasite loads and organ damage [29].
There is plenty of evidence indicating that $T$. gondii actively interferes with host cell signaling during intracellular infection [6,30-32]. The signal transducer and activator of transcription (STAT) signaling pathway as well as NF- $\mathrm{B}$ signaling cascade have emerged as two major targets of exploitation by $T$. gondii $[6,33]$. Previous data revealed that a single ROP16 polymorphism at position 503 determines the strain difference with respect to STAT3 activation [34], which negatively affects NF-kB signal pathway, leads to suppression of IL-12, and 
induces differentiation of the infected cells into immune suppressive macrophages $[35,36]$. Besides, the type I/III ROP16 is capable of directly catalyzing tyrosine phosphorylation of STAT6 $[3,33,37,38]$, and responsible for M2 polarization [3]. Here, we found that although sharing the same 503Leu in ROP16 (additional files show this in more detail [see Additional file 1 and Additional file 2]) and inducing activation of p-STAT3 resemble RH strain, TgCtwh3 and TgCtWh6 isolates trigger notable difference in phosphorylation of STAT6, and promote disparate bias of macrophages. Our results suggest that in addition to ROP16, other potential effector molecules (e.g. ROP18, data not shown) might be involved in the activation of macrophage polarization in China 1 isolates.

On the other hand, macrophages infected with TgCtwh6 give rise to a high production of IL-12p40 and $\mathrm{NO}$, enhanced expression of iNOS on mRNA and protein levels (Figure 4), as well as phospho-ІкB $\alpha$ and nuclear translocation of NF-kB p65 (Figure 6). Therefore, it is reasonable to conclude that $\mathrm{TgCtwh} 6$ strain activates NF-кB pathway and induces M1-like cells. Thus, TgCtwh6 possesses the features similar to the type II strains (e.g., PRU), such as less virulence and encystation, induction of high IL-12p40 and activation of NF$\kappa B$ pathway, in spite of alteration of amino acid at 503 of ROP16 and activation of STAT3. Further studies are needed to disclose the polymorphism GRA15 of TgCtwh6 isolate and the polymorphism-associated pathogenesis based on the evidence that type II GRA15 determines M1 polarization [3].

In conclusion, the results indicate that both TgCtwh3 and $\mathrm{TgCtWh} 6$ isolates may elicit macrophage-biased responses in different pathways. TgCtwh3 induces activated macrophages resembling M2 through phosphorylation of STAT6, whereas TgCtwh6 activates NF- $\mathrm{kB}$ pathway and promotes infected macrophages to skew to a M1 phenotype. The demonstration of different phenotypes among China 1 isolates may be due to the fact that restriction fragment length polymorphism (RFLP) analysis, although valuable for structural population approach, captures only a fraction but not all of the genetic diversity at a given locus. Hence, differences in virulence, cytokine induction and macrophage activation of both isolates make more thorough genetic analysis and analysis of other effectors essential, which may help elucidate the mechanism of the immune response during acute and latent infection with Toxoplasma and better understand the influence of strain genotype on human toxoplasmosis.

\section{Conclusions}

The present study demonstrated that macrophages infected with ToxoDB \#9 strains of different virulence were polarized toward different activation states through disparate signaling pathway, which may ultimately influence the ability of Toxoplasma to establish a chronic infection. To our knowledge, this is the first report of macrophage activation induced by ToxoDB \#9 parasites isolated from China. Further studies on other effector molecules may help understand how ToxoDB \#9 strains regulate immune responses and provide important clues to disclosure of their essential variance.

\section{Additional files}

Additional file 1: Supplemental methods and the legend of Figure S1. Additional file 2: Sequence alignment of ROP16 alleles.

\section{Abbreviations}

M $\varphi$ : Macrophage; M1: Classically activated macrophage; M2: Alternatively activated macrophage; BMM $\varphi$ : Bone marrow-derived macrophage; PECs: Peritoneal exudate cells; iNOS: Inducible nitric oxide synthase; PD-L: Programmed death ligand; GRA: Dense granule antigen; NO: Nitric oxide; MFI: Mean fluorescence intensity; ROP: Rhoptry protein; STAT: Signal transducer and activator of transcription.

\section{Competing interests}

The authors declared that they have no competing interests.

\section{Authors' contributions}

$J$ LS, AMZ, QS, ZRL, and QS conceived and designed the study, and critically revised the manuscript. $A M Z, Q S, M L$ and $H C$ performed the experiments and drafted the manuscript. XCX, YW, DYC, LY, and JD participated in the analysis and interpretation of data. YHC and QLL performed the statistical analysis. All authors have read and approved the final manuscript.

\section{Acknowledgements}

This work was financially supported by Natural Basic Research Program of China (973 Program, No. 2010 CB530001), the Natural Science Foundation of Anhui Province, China (NO. 1208085 MH165), and the Education Department Research Program of Anhui Province, China (NO. KJ2012A145). The authors are thankful to Prof. Wen-Qi Liu at Faculty of Medicine, Huazhong University of Science and Technology, and Prof. Wen-Jian Song at Department of Parasitology of Jianghan University, for their assistance in collecting animals for parasite isolation.

\section{Author details}

${ }^{1}$ Anhui Provincial Laboratories of Pathogen Biology and Zoonoses, Department of Microbiology and Parasitology, Anhui Medical University, Hefei, Anhui, China. ${ }^{2}$ Central Laboratory of Affiliated Provincial Hospital of Anhui Medical University, Hefei, Anhui, China. ${ }^{3}$ Department of Immunology, Anhui Medical Universty, Hefei, Anhui, China. ${ }^{4}$ Department of Biochemistry and Molecular Biology, Anhui Medical University, Hefei, China. ${ }^{5}$ Center for Parasitic Organisms, State Key Laboratory of Biocontrol, School of Life Sciences and Key Laboratory of Tropical Diseases and Control of the Ministry of Education, Sun Yat-Sen University, Guangzhou, Guangdong, China. ${ }^{6}$ Department of Pathogen Biology, Nanjing Medical University, Nanjing, China.

Received: 24 September 2013 Accepted: 22 October 2013 Published: 26 October 2013

\section{References}

1. Hill D, Dubey JP: Toxoplasma gondii: transmission, diagnosis and prevention. Clin Microbiol Infect 2002, 8:634-640.

2. di Carlo P, Romano A, Casuccio A, Cillino S, Schimmenti MG, Mancuso G, la Chiusa S, Novara V, Ingrassia D, Li Vecchi V, Trizzino M, Titone L: Investigation and management of Toxoplasma gondii infection in pregnancy and infancy: a prospective study. Acta Pharmacol Sin 2011, 32:1063-1070.

3. Jensen KD, Wang Y, Wojno ED, Shastri AJ, Hu K, Cornel L, Boedec E, Ong YC, Chien YH, Hunter CA, Boothroyd JC, Saeij JP: Toxoplasma polymorphic 
effectors determine macrophage polarization and intestinal inflammation. Cell Host Microbe 2011, 9:472-483.

4. Mosser DM, Edwards JP: Exploring the full spectrum of macrophage activation. Nat Rev Immunol 2008, 8:958-969.

5. Stein M, Keshav S, Harris N, Gordon S: Interleukin 4 potently enhances murine macrophage mannose receptor activity: a marker of alternative immunologic macrophage activation. J Exp Med 1992, 176:287-292.

6. Rosowski EE, Lu D, Julien L, Rodda L, Gaiser RA, Jensen KD, Saeij JP: Strain-specific activation of the NF-kappaB pathway by GRA15, a novel Toxoplasma gondii dense granule protein. J Exp Med 2011, 208:195-212.

7. Boyle JP, Rajasekar B, Saeij JP, Ajioka JW, Berriman M, Paulsen I, Roos DS, Sibley LD, White MW, Boothroyd JC: Just one cross appears capable of dramatically altering the population biology of a eukaryotic pathogen like Toxoplasma gondii. Proc Natl Acad Sci U S A 2006, 103:10514-10519.

8. Sibley LD, Ajioka JW: Population structure of Toxoplasma gondii: clonal expansion driven by infrequent recombination and selective sweeps. Annu Rev Microbiol 2008, 62:329-351.

9. Saeij JP, Boyle JP, Coller S, Taylor S, Sibley LD, Brooke-Powell ET, Ajioka JW, Boothroyd JC: Polymorphic secreted kinases are key virulence factors in toxoplasmosis. Science 2006, 314:1780-1783.

10. Khan A, Dubey JP, Su C, Ajioka JW, Rosenthal BM, Sibley LD: Genetic analyses of atypical Toxoplasma gondii strains reveal a fourth clonal lineage in North America. Int J Parasitol 2011, 41:645-655.

11. Mercier A, Devillard S, Ngoubangoye B, Bonnabau H, Banuls AL, Durand P, Salle B, Ajzenberg D, Darde ML: Additional haplogroups of Toxoplasma gondii out of Africa: population structure and mouse-virulence of strains from Gabon. PLoS Negl Trop Dis 2010, 4:e876.

12. Chen ZW, Gao JM, Huo XX, Wang L, Yu L, Halm-Lai F, Xu YH, Song WJ, Hide G, Shen JL, Lun ZR: Genotyping of Toxoplasma gondii isolates from cats in different geographic regions of China. Vet Parasitol 2011, 183:166-170.

13. Jiang HH, Huang SY, Zhou DH, Zhang XX, Su C, Deng SZ, Zhu XQ: Genetic characterization of Toxoplasma gondii from pigs from different localities in China by PCR-RFLP. Parasit Vectors 2013, 6:227.

14. Lin W, Hua-Wei C, Kai-Quan H, Yuan-Hong X, Yong-Nian L, Jian D, Li Y, Qing-Li L, Wei W, Ling J, Ji-Long S: Toxoplasma gondii prevalence in food animals and rodents in different regions of China: isolation, genotyping and mouse pathogenicity. Parasit Vectors 2013, 6:273.

15. Chen J, Li ZY, Zhou DH, Liu GH, Zhu XQ: Genetic diversity among Toxoplasma gondii strains from different hosts and geographical regions revealed by sequence analysis of GRA5 gene. Parasit Vectors 2012, 5:279.

16. Boothroyd JC, Grigg ME: Population biology of Toxoplasma gondii and its relevance to human infection: do different strains cause different disease? Curr Opin Microbiol 2002, 5:438-442.

17. Luder CG, Algner M, Lang C, Bleicher N, Gross U: Reduced expression of the inducible nitric oxide synthase after infection with Toxoplasma gondii facilitates parasite replication in activated murine macrophages. Int J Parasitol 2003, 33:833-844.

18. Corraliza IM, Campo ML, Soler G, Modolell M: Determination of arginase activity in macrophages: a micromethod. J Immunol Methods 1994, 174:231-235.

19. Seipel D, Ribeiro-Gomes FL, Barcelos MW, Ramalho AV, Kanashiro MM, Kipnis TL, Arnholdt AC: Monocytes/macrophages infected with Toxoplasma gondii do not increase co-stimulatory molecules while maintaining their migratory ability. APMIS 2009, 117:672-680.

20. Lang C, Algner M, Beinert N, Gross U, Luder CG: Diverse mechanisms employed by Toxoplasma gondii to inhibit IFN-gamma-induced major histocompatibility complex class II gene expression. Microbes Infect 2006 8:1994-2005.

21. Luder CG, Lang T, Beuerle B, Gross U: Down-regulation of MHC class II molecules and inability to up-regulate class I molecules in murine macrophages after infection with Toxoplasma gondii. Clin Exp Immunol 1998, 112:308-316.

22. Wang L, Chen H, Liu D, Huo X, Gao J, Song X, Xu X, Huang K, Liu W, Wang $Y$, Lu F, Lun ZR, Luo Q, Wang X, Shen J: Genotypes and mouse virulence of Toxoplasma gondii isolates from animals and humans in China. PLoS One 2013, 8:e53483.

23. Morgado P, Ong YC, Boothroyd JC, Lodoen MB: Toxoplasma gondii induces B7-2 expression through activation of JNK signal transduction. Infect Immun 2011, 79:4401-4412.

24. Murray PJ: Macrophages as a battleground for Toxoplasma pathogenesis. Cell Host Microbe 2011, 9:445-447.
25. El Kasmi KC, Qualls JE, Pesce JT, Smith AM, Thompson RW, Henao-Tamayo M, Basaraba RJ, Konig T, Schleicher U, Koo MS, Kaplan G, Fitzgerald KA, Tuomanen El, Orme IM, Kanneganti TD, Bogdan C, Wynn TA, Murray PJ: Toll-like receptor-induced arginase 1 in macrophages thwarts effective immunity against intracellular pathogens. Nat Immuno/ 2008, 9:1399-1406.

26. Li Z, Zhao ZJ, Zhu XQ, Ren QS, Nie FF, Gao JM, Gao XJ, Yang TB, Zhou WL, Shen JL, Wang Y, Lu FL, Chen XG, Hide G, Ayala FJ, Lun ZR: Differences in iNOS and arginase expression and activity in the macrophages of rats are responsible for the resistance against T. gondii infection. PLOS One 2012, 7:e35834.

27. Robben PM, Mordue DG, Truscott SM, Takeda K, Akira S, Sibley LD: Production of IL-12 by macrophages infected with Toxoplasma gondii depends on the parasite genotype. J Immunol 2004, 172:3686-3694.

28. Henrik L, Vutova PP, Adams WC, Karin L, Antonio B: The Toxoplasma gondii-shuttling function of dendritic cells is linked to the parasite genotype. Infect Immun 2009, 77:1679-1688.

29. Mordue DG, Monroy F, La Regina M, Dinarello CA, Sibley LD: Acute toxoplasmosis leads to lethal overproduction of Th1 cytokines. J Immunol 2001, 167:4574-4584.

30. Butcher BA, Kim L, Panopoulos AD, Watowich SS, Murray PJ, Denkers EY: IL-10-independent STAT3 activation by Toxoplasma gondii mediates suppression of IL-12 and TNF-alpha in host macrophages. J Immunol 2005, 174:3148-3152.

31. Peixoto L, Chen F, Harb OS, Davis PH, Beiting DP, Brownback CS, Ouloguem $D$, Roos DS: Integrative genomic approaches highlight a family of parasite-specific kinases that regulate host responses. Cell Host Microbe 2010, 8:208-218.

32. Whitmarsh RJ, Gray CM, Gregg B, Christian DA, May MJ, Murray PJ, Hunter CA: A critical role for SOCS3 in innate resistance to Toxoplasma gondii. Cell Host Microbe 2011, 10:224-236.

33. Butcher BA, Fox BA, Rommereim LM, Kim SG, Maurer KJ, Yarovinsky F, Herbert DR, Bzik DJ, Denkers EY: Toxoplasma gondii rhoptry kinase ROP16 activates STAT3 and STAT6 resulting in cytokine inhibition and arginase-1-dependent growth control. PLOS Pathog 2011, 7:e1002236.

34. Yamamoto M, Standley DM, Takashima S, Saiga H, Okuyama M, Kayama H, Kubo E, Ito H, Takaura M, Matsuda T, Soldati-Favre D, Takeda K: A single polymorphic amino acid on Toxoplasma gondii kinase ROP16 determines the direct and strain-specific activation of Stat3. J Exp Med 2009, 206:2747-2760.

35. Mordue DG, Sibley LD: A novel population of Gr-1+-activated macrophages induced during acute toxoplasmosis. J Leukoc Biol 2003, 74:1015-1025

36. Trinchieri G: Interleukin-12 and the regulation of innate resistance and adaptive immunity. Nat Rev Immunol 2003, 3:133-146.

37. Ong YC, Reese ML, Boothroyd JC: Toxoplasma rhoptry protein 16 (ROP16) subverts host function by direct tyrosine phosphorylation of STAT6. J Biol Chem 2010, 285:28731-28740.

38. Saeij JP, Coller S, Boyle JP, Jerome ME, White MW, Boothroyd JC: Toxoplasma co-opts host gene expression by injection of a polymorphic kinase homologue. Nature 2007, 445:324-327.

doi:10.1186/1756-3305-6-308

Cite this article as: Zhang et al:: Comparative studies of macrophagebiased responses in mice to infection with Toxoplasma gondii ToxoDB \#9 strains of different virulence isolated from China. Parasites \& Vectors $20136: 308$ 